\title{
Allograft Inflammatory Factor-1 Mediates Macrophage-Induced Impairment of Insulin Signaling in Adipocytes
}

\author{
Jingqi Ren Yaqiu Lin Junni Tang Hua Yue Yanying Zhao \\ College of Life Science and Technology, Southwest University for Nationalities, Chengdu, PR China
}

\section{Key Words}

Allograft inflammatory factor-1 • AIF-1 1 Inflammation • Insulin signaling • Adipocyte

\begin{abstract}
Background/Aims: Allograft inflammatory factor-1 (AIF-1) is an inflammatory cytokine produced mainly by macrophages within human white adipose tissue. Its expression is increased in obese subjects and positively correlated with insulin resistance. The purpose of this study is to characterize the regulatory role of AIF-1 in insulin signaling of adipocyte. Methods: AIF-1 was over-expressed via transfection of AIF-1 cDNA into murine RAW 264.7 macrophages, and the constitutive expression of AIF- 1 was decreased via transfection of targeting siRNA. Murine 3T3L1 adipocytes were treated with macrophage-conditioned medium or AIF-1 protein. Intracellular lipid accumulation was assayed by oil red $O$ stain. Reactive oxygen species production was determinated by a flow cytometer and adipokine secretion was measured with ELISA. Glucose uptake was detected using the glucose oxidase method and insulinsignal-transduction related molecules were analyzed by Western blot. Results: Short term (48 h) AIF-1 treatment slightly promoted intracellular lipid storage in differentiating 3T3L1 cells. The protein stimulated reactive oxygen species production, provoked TNF $\alpha$, IL6, resistin, but suppressed adiponectin release and insulin-stimulated glucose uptake both under normal basal and insulin resistance conditions. Furthermore, AIF-1 induced NF-kB activation, inhibited PPARy expression, GLUT4 translocation to plasma membrane and Akt phosphorylation. Conclusion: Macrophage-derived AIF-1 up-regulated reactive oxygen species production, adipokine TNF $\alpha$, IL6, resistin release, and inhibited adiponectin secretion. Moreover, it suppressed insulin-stimulated glucose uptake by down-regulating insulin signaling. Thus, AIF-1 could be related to obesity-related diseases.

\section{Introduction}

Obesity is a major risk factor for the development of insulin resistance, and the progression of diabetes, cardiovascular disease, and other comorbidities [1]. It is considered as a state of chronic low-grade systemic inflammation due to infiltration of activated macrophages in adipose tissue $[2,3]$. Numerous studies have stressed that chronic inflammation link


excess fat to metabolic disorders [3, 4]. Adipose tissue macrophages produce a number of pro-inflammatory cytokines that significantly alter adipocyte function, inducing lipid accumulation [5], inflammatory responses [6] and decreasing insulin sensitivity [7]. Identification of the major factors that mediate detrimental effects of adipocyte may offer potential therapeutic targets.

Allograft inflammatory factor-1 (AIF-1) is a calcium-binding inflammation related scaffold protein mainly produced by immune cells [8]. It was firstly identified in activated macrophages in rat cardiac allografts with chronic rejection (GenBank accession number U17919) [9]. Several other molecules such as daintain [10], ionized $\mathrm{Ca}^{2+}$-binding adapter (Iba1) [11] and microglia response factor 1 (MRF-1) [12] are homologues of AIF-1. AIF-1 has been recognized as a crucial molecular for the survival and pro-inflammatory activity of macrophages $[13,14]$. Thus, it is involved in inflammation and immune responses associated with vasculopathy [15], autoimmune diseases [16], and central nervous system (CNS) injury [17] et al.

Recently, several reports indicated the involvement of AIF-1 in obesity. First, a single nucleotide polymorphism in the AIF-1 gene region was related to body weight [18]. Second, AIF-1 $1^{-/}$mice were protected from diet induced obesity [19]. Third, AIF-1 was suggested as a novel adipokine produced mainly by macrophages within human white adipose tissue. Its expression was increased in obese subjects and positively correlated with insulin resistance [20]. Based on these findings, the role of AIF-1 in macrophage-induced signaling sensitivity of adipocytes was investigated in the present study using murine RAW264.7 macrophages and 3T3L1 adipocytes.

\section{Materials and Methods}

\section{Vector construction and transfection}

The pcDNA3.1-AIF1 plasmid was constructed by subcloning the cDNA of murine AIF1 (GenBank accession number: AB013745.1) into pcDNA3.1(+) at the sites of BamH1 and Xho1 (underlined sequences) with primers: sense, 5'-CGGGATCCGCCACCATGAGCCAAAG $-3^{\prime}$ and antisense, 5'-CCGCTCGAGTCAGGGCAGCTCGGAG-3'. Insertion of cDNA was verified by DNA sequencing. In addition, three short interference RNAs (siRNAs) for AIF-1 were synthesized targeted (sequence 1, GCCTTCAAGGTGAAGTACA, siAIF1-1; sequence 2, CCTAGAGCTGAAGAGATTA, siAIF1-2; or sequence 3, CCATCTTGAGAATGATTCT, siAIF1-3) with negative control (target sequence: TTCTCCGAACGTGTCACGT, siRNA) and interference RNA for GAPDH (target sequence: CACTCAAGATTGTCAGCAA, GAPDH420). A murine macrophage cell line, RAW264.7, was obtained from American Type Culture Collection (ATCC, Manassas, VA, USA). The cells were activated by lipopolysaccharide and cultured in Dulbecco's modified Eagle's medium (DMEM, Gibco, NY, USA) supplemented with 10\% fetal bovine serum (FBS), $100 \mathrm{U} / \mathrm{mL}$ penicillin and $100 \mathrm{U} / \mathrm{mL}$ streptomycin at $37^{\circ} \mathrm{C}$ in the humidified atmosphere of $5 \% \mathrm{CO}_{2}$. Then, the cell culture medium was replaced to serum-free DMEM and transfection was performed with a Sofast (Xiamen, Fujian, China) according to the manufacturer's instructions. $48 \mathrm{~h}$ later, the medium was harvested by centrifuging at $350 \mathrm{~g}$ for $10 \mathrm{~min}$, and filtered through a $0.22 \mu \mathrm{m}$ filter.

\section{Oil Red $O$ assay}

Murine 3T3L1 pre-adipocytes were also obtained from ATCC and grown in DMEM with 10\% FBS and $1 \%$ penicillin/streptomycin at $37{ }^{\circ} \mathrm{C}$ in the humidified atmosphere of $5 \% \mathrm{CO}_{2}$. The pre-adipocytes were differentiated into adipocytes as we previously described [21]. Briefly, 2 days after confluence (day 0 ), cells were induced to differentiate by DMEM supplemented with $10 \% \mathrm{FBS}, 0.5 \mathrm{mmol} / \mathrm{L} 3$-isobutyl-1methyl-xanthine, $0.25 \mu \mathrm{mol} / \mathrm{L}$ dexamethasone and $10 \mu \mathrm{g} / \mathrm{mL}$ insulin (MDI, Sigma, St. Louis, MO, USA) for another 2 days. On the $4^{\text {th }}$ day, cells were transferred into macrophage-conditioned medium and $10 \mu \mathrm{g} / \mathrm{mL}$ insulin for two more days. Intracellular lipid accumulation of 3T3L1 adipocytes was monitored by the visual appearance of fat droplets in the cells and oil red 0 stain. The integrated optical density (IOD) of the lipid droplets was used to quantify the lipid accumulation [22].

\section{KARGER}




\section{Cellular Physiology Cell Physiol Biochem 2018;47:403-413

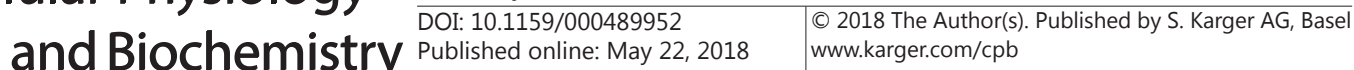 \\ Ren et al.: Mediates Insulin Signaling in Adipocytes}

\section{Measurement of reactive oxygen species and adipokine release}

Murine 3T3L1 pre-adipocytes were fully differentiated into adipocytes using a standard protocol [21]. Specifically, 2 days after the cells reached confluence, they were incubated in the differentiation medium (DMEM supplemented with 10\% FBS and adipogenic cocktail MDI) for 2 days. Then, the cells were maintained in DMEM containing $10 \% \mathrm{FBS}$ and $10 \mu \mathrm{g} / \mathrm{mLinsulin}$ for another 2 days, and replenished with DMEM containing $10 \%$ FBS until full differentiation into adipocytes. Thereafter, the medium was replaced with macrophageconditioned medium or $0,0.1,1,10 \mathrm{nmol} / \mathrm{L}$ AIF-1 [16] in DMEM supplemented with $10 \%$ FBS for 2 days. The cells were harvested by centrifuging at $500 \mathrm{~g}$ for $5 \mathrm{~min}$ and lysed. The reactive oxygen species production in 3T3L1 adipocytes was measured with cellular reactive oxygen species detection assay kit (Sigma, St. Louis, MO, USA), and analyzed by a flow cytometer (Beckman Coulter FC500, Brea, CA). The secretion concentrations of tumor necrosis factor-alpha (TNF- $\alpha$ ), interleukin 6 (IL6), resistin and adiponectin from adipocytes and the basal levels of TNF $\alpha$, IL6 in macrophage-conditioned medium were determined using the corresponding ELISA kits (R\&D Systems, Abingdon, UK).

\section{Glucose consumption}

Glucose consumption was examined as previously described [23]. Differentiated 3T3L1 adipocytes were exposed for $18 \mathrm{~h}$ to DMEM containing 10\% FBS and either $5 \mathrm{mmol} / \mathrm{L}$ or $25 \mathrm{mmol} / \mathrm{L}$ glucose, with or without $0.6 \mathrm{nmol} / \mathrm{L}$ insulin, as indicated. Then, the cells were incubated in macrophage-conditioned medium or $0,0.1,1,10 \mathrm{nmol} / \mathrm{L}$ AIF-1 in DMEM with $10 \%$ FBS for $48 \mathrm{~h}$. Before assay, the cells were stimulated with $100 \mathrm{nmol} / \mathrm{L}$ insulin for $30 \mathrm{~min}$ and the concentration of glucose in the culture medium was analyzed using the glucose oxidase method.

\section{Western blot}

Western blot was employed to detect the expression levels of AIF-1 in RAW264.7 cells at the $48^{\text {th }} \mathrm{h}$ after transfection and to measure nuclear factor kappa B (NF- $\mathrm{kB}$ ) p65, AKT phosphorylation, peroxisome proliferator-activated receptor- $\gamma$ (PPAR $\gamma$ ) expression, and insulin-responsive glucose transporter 4 (GLUT 4) translocation in differentiated 3T3L1 adipocytes after macrophage-conditioned medium or AIF-1 protein treatment. Total cellular proteins were prepared with lysis buffer containing protease and phosphatase inhibitors (Sigma, St. Louis, MO, USA). The membrane proteins were isolated and extracted with a plasma membrane protein extraction kit (No. PAB180006, Bioswamp, China). Briefly, $5 \times 10^{7} 3 \mathrm{~T} 3 \mathrm{~L} 1$ adipocytes were washed, re-suspended and homogenized in RIPA lysis buffer containing protease and phosphatase inhibitors with a mortar and pestle. Then the resulting homogenates were centrifuged at $14000 \mathrm{~g}$ for $30 \mathrm{~min}$ at 4 ${ }^{\circ} \mathrm{C}$. After the supernatant was removed, the pellets (membrane debris) were repeatedly homogenized and extensively washed to eliminate the soluble components. Thereafter, the membrane pellets were lysed with solution B in the kit and intermittently oscillated with a vortex oscillator for $30 \mathrm{~min}$ at $4{ }^{\circ} \mathrm{C}$, then centrifuged at $14,000 \mathrm{~g}$ for $5 \mathrm{~min}$ at $4{ }^{\circ} \mathrm{C}$. The supernatant was the extracted plasma membrane proteins. Protein concentrations were determined using a Pierce ${ }^{\mathrm{TM}}$ BCA Protein Assay Kit (Thermo Scientific, Waltham, MA, USA). The proteins were separated on SDS-PAGE, and transferred to nitrocellulose membranes (Whatman, London, UK). Then the membranes were blocked with TBST (20 mmol/L Tris-HCl, pH 7.6, 0.14 mol/L NaCl, $0.1 \%$ Tween 20 ) containing $5 \% \mathrm{w} / \mathrm{v}$ bovine serum albumin for $1 \mathrm{~h}$ at room temperature. After that, the membranes were incubated overnight at $4{ }^{\circ} \mathrm{C}$ with the specific primary antibodies against AIF-1 (1:2000,

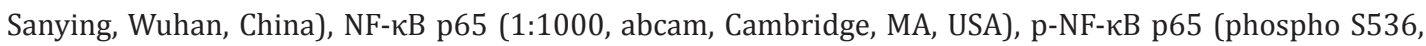
1:1000, abcam, Cambridge, MA, USA), GLUT 4 (1:1000, abcam, Cambridge, MA, USA), AKT (1:1000, abcam, Cambridge, MA, USA), p-AKT (phospho S473, 1:1000, abcam, Cambridge, MA, USA), GAPDH (1:1000, Sanying, Wuhan, China). Membranes were washed with TBST thoroughly before the corresponding horseradish peroxidase (HRP)-conjugated secondary antibodies were treated. Specific immune complexes were detected by chemiluminescence (West Pico kit, Pierce, Loughborough, UK) and scanned using ChemiDoc ${ }^{\mathrm{TM}}$ $\mathrm{XRS}^{+}$(Bio-Rad, Hercules, CA, USA).

\section{Statistical analysis}

Data were expressed as means \pm standard error of the mean (SEM). Data were analyzed statistically using SPSS Statistics V17.0 software and the significance of the differences between the corresponding groups was determined by ANOVA followed by one tailed multiple t-tests. Value of $P<0.05$ was considered as statistical significance. 


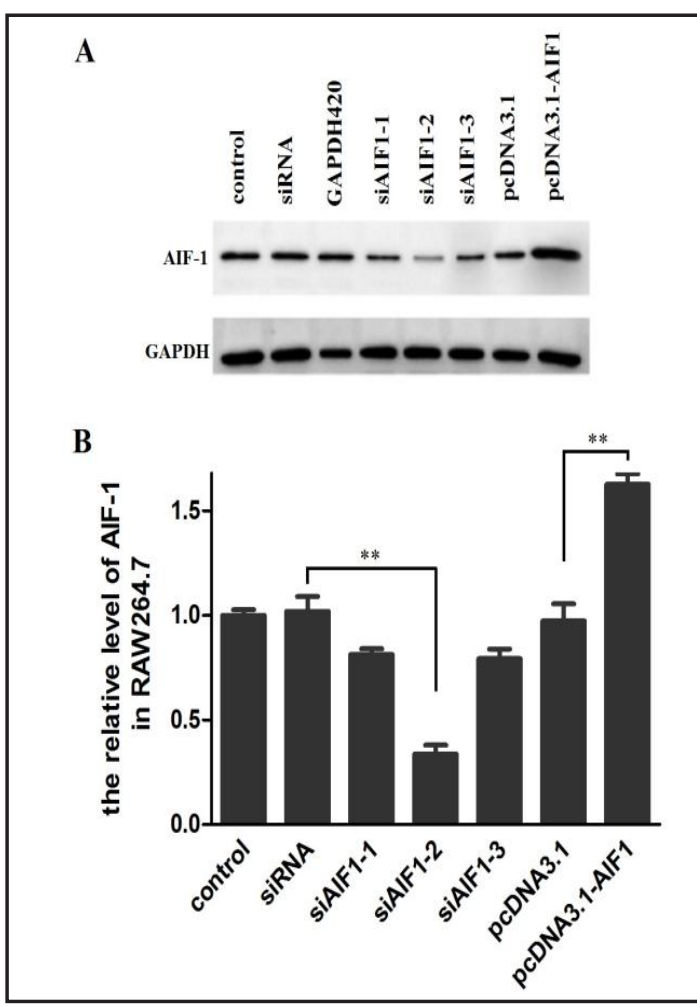

Fig. 1. AIF-1 expression in murine RAW264.7 macrophage cells. A. Western blot analysis of AIF-1 in RAW264.7 macrophage cells. control, the baseline expression of AIF-1 in Raw 264.7 macrophage cells; RAW264.7 cells were tranfected with: siRNA, negative control (target sequence TTCTCCGAACGTGTCACGT); GAPDH420: interference RNA for GAPDH (target sequence CACTCAAGATTGTCAGCAA); siAIF1-1, short interference RNA (target sequence GCCTTCAAGGTGAAGTACA); siAIF1-2, short interference RNA (target sequence CCTAGAGCTGAAGAGATTA); siAIF1-3, short interference RNA (target sequence CCATCTTGAGAATGATTCT); pcDNA3.1, plasmid pcDNA3.1; pcDNA3.1-AIF1, plasmid pcDNA3.1 subcloned with the cDNA of murine AIF1(GenBank accession number: AB013745.1). B. The level of AIF-1 expression in RAW264.7 macrophage cells. The level of AIF-1 in siAIF1-2 was significantly lower than that in siRNA, the level of AIF-1 in pcDNA3.1-AIF1 was significantly higher than that in pcDNA3.1. Data were the mean \pm SEM of three independent experiments. $\left({ }^{* *} \mathrm{P}<0.01\right)$.

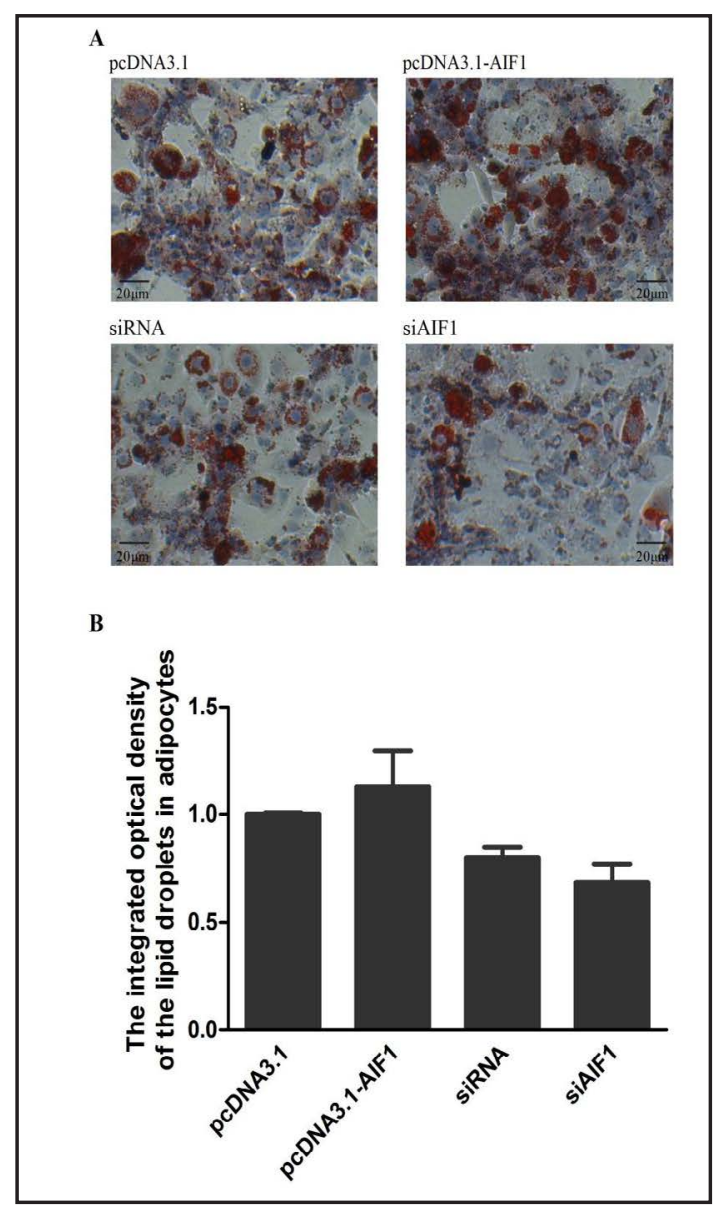

Fig. 2. Oil red 0 stain of 3T3L1 differentiating adipocytes. 3T3L1 pre-adipocytes were induced to differentiate by DMEM supplemented with $10 \%$ fetal bovine serum, $0.5 \mathrm{mmol} / \mathrm{L}$ 3-isobutyl-1-methylxanthine, $0.25 \mu \mathrm{mol} / \mathrm{L}$ dexamethasone and $10 \mu \mathrm{g} /$ $\mathrm{mL}$ insulin for 2 days. Then the cells were transferred into macrophage-conditioned medium and $10 \mu \mathrm{g} /$ $\mathrm{mL}$ insulin for another 2 days. Intracellular lipid accumulation was monitored by oil red 0 stain. A. image of lipid droplets in 3T3L1 adipocytes stained by oil red 0 . B. the integrated optical density of the lipid droplets. Data were the mean \pm SEM of three independent experiments.

\section{Results}

AIF-1 over-expression and interference

Lorente-Cebrián and colleagues reported that AIF-1 was secreted in a time dependent manner from white adipose tissue and the major source of AIF-1 was white adipose tissue resident macrophages [20]. In order to investigate the role of AIF-1 in obesity, the level of 
AIF-1 expression was up-regulated via transfection of the gene into RAW264.7 (pcDNA3.1-AIF1 in Fig. 1), and decreased via transfection of siRNA against AIF1(siAIF1-1, siAIF1-2 and siAIF1-3 in Fig. 1). The level of AIF-1 in siAIF1-2 was significantly lower than that in siRNA, the level of AIF-1 in pcDNA3.1AIF1 was significantly higher than that in pcDNA3.1.Therefore, the serum free DMEM media of RAW264.7 macrophage in pcDNA3.1, pcDNA3.1-AIF1, siRNA and siAIF1-2 were harvested for the following experiment.

\section{Oil red $O$ assay}

After 3T3-L1 pre-adipocytes were induced with MDI, they were incubated in macrophage-conditioned medium supplemented with $10 \%$ FBS for 2 days and intracellular triglyceride accumulation was monitored by oil red 0 stain as shown in Fig. 2. Although the cells had not been fully differentiated into adipocytes. There was a little more intracellular lipid accumulation in 3T3-L1 cells treated with pcDNA3.1AIF1 macrophage-conditioned medium. While AIF-1 expression down-regulation slightly inhibited intracellular triglyceride accumulation in 3T3L1 cells. Thus, short term (48 h) AIF-1 treatment slightly promoted intracellular lipid accumulation in differentiating 3T3L1 cells.

Reactive oxygen species production and adipokine secretion

In addition, the effect of macrophageconditioned medium on reactive oxygen species production, TNF $\alpha$, IL6, resistin and adiponectin secretion was assayed. The results revealed that AIF-1 upregulation increased $\mathrm{TNF} \alpha(1.26 \pm 0.01$ fold, $P<0.001$, Fig. 3A), IL6 (1.45 \pm 0.01 fold, $P<0.001$, Fig. 3B), resistin $(1.52 \pm 0.02$ fold, $P<0.001$, Fig. $3 \mathrm{C}$ ) release and reactive oxygen species production $(1.65 \pm 0.09$ fold, $P<0.01$, Fig. 3E), while decreased adiponectin secretion $(0.69 \pm 0.01$ fold, $P$ $<0.001$, Fig. 3D). At the same time, AIF-1 interference inhibited $\mathrm{TNF} \alpha(0.84 \pm 0.01$ fold, $P<0.001$, Fig. 3A), IL6 $(0.79 \pm 0.02$ fold, $P<0.001$, Fig. 3B), resistin $(0.84 \pm 0.01$ fold, $P<0.001$, Fig. $3 C)$ release and reactive oxygen species production $(0.88 \pm 0.12$ fold, Fig. $3 \mathrm{E}$ ), but promoted adiponectin secretion ( $1.24 \pm 0.02$ fold, $P<0.001$, Fig. 3D). In line with this,
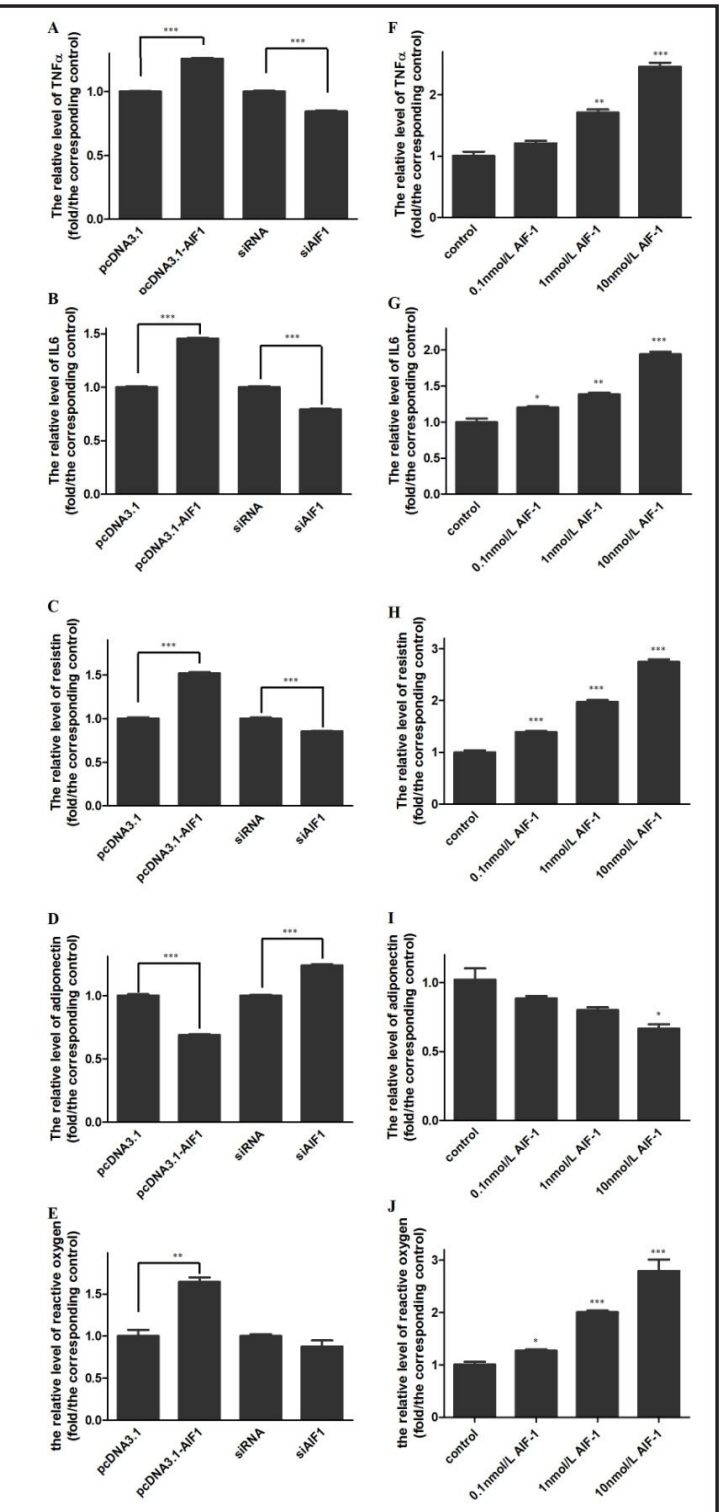

Fig. 3. Reactive oxygen species production and adipokine release in 3T3L1 adipocytes. 3T3L1 adipocytes were treated with macrophage-conditioned medium (A, B, C, D, E) or 0, 0.1, 1, $10 \mathrm{nmol} / \mathrm{L} \mathrm{AIF-1} \mathrm{in} \mathrm{DMEM} \mathrm{(F,}$ G, H, I, J) supplemented with $10 \%$ fetal bovine serum for 2 days. The reactive oxygen species production in 3T3L1 adipocytes was measured with cellular reactive oxygen species detection assay kit. The secretion concentrations of tumor necrosis factor-alpha (TNF- $\alpha$ ), interleukin 6 (IL6), resistin and adiponectin from adipocytes were determined using the corresponding ELISA kits. Data were the mean \pm SEM of three independent experiments. ( $\left.{ }^{*} \mathrm{P}<0.05,{ }^{* *} \mathrm{P}<0.01,{ }^{* * *} \mathrm{P}<0.001\right)$. 


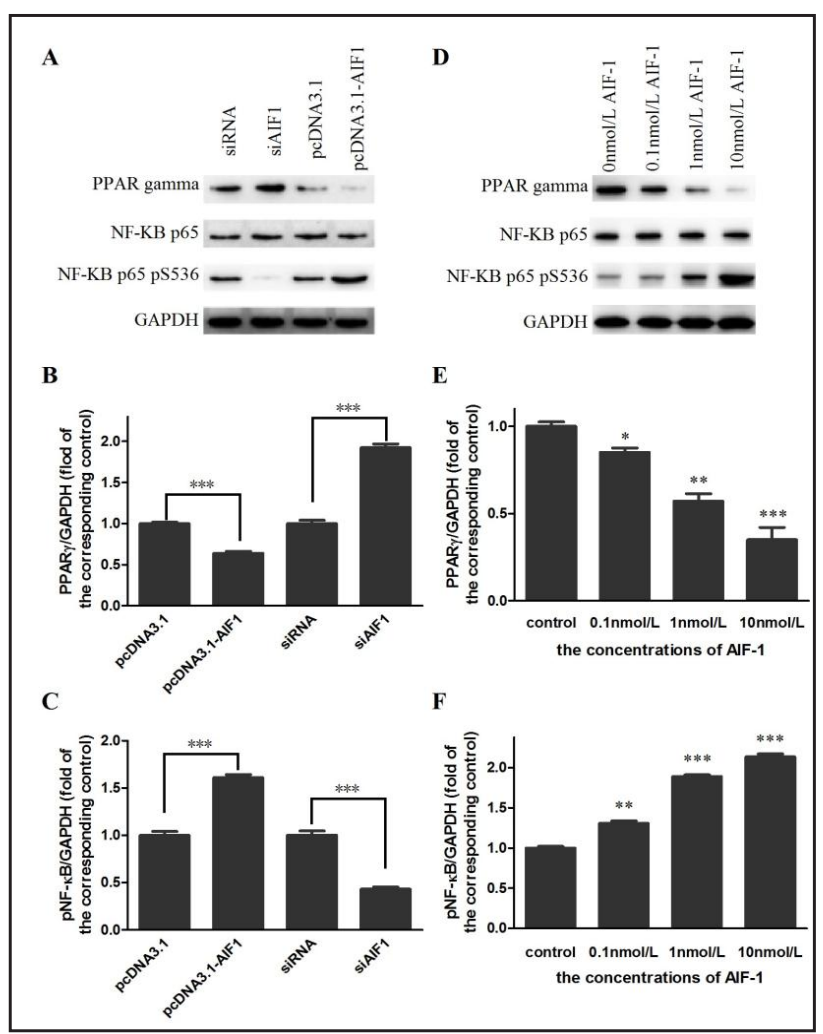

Fig. 4. The effect of AIF-1 on peroxisome proliferatoractivated receptor- $\gamma$ (PPAR $\gamma$ ) expression and nuclear factor kappa B (NF- $\mathrm{B}$ ) phosphorylation. 3T3L1 adipocytes were incubated with macrophage-conditioned medium (A, B, C) or 0, 0.1, 1, $10 \mathrm{nmol} / \mathrm{L} \mathrm{AIF-1} \mathrm{in} \mathrm{DMEM} \mathrm{(D,} \mathrm{E,} \mathrm{F)} \mathrm{supplemented}$ with $10 \%$ fetal bovine serum for 2 days. PPAR $\gamma$ expression and NF- $\kappa B$ phosphorylation (S536) were analyzed by Western blot. Data were the mean \pm SEM of three independent experiments. $(* * * \mathrm{P}<0.001)$.

AIF-1 protein also up-regulated TNF $\alpha$ (Fig. 3F), IL6 (Fig. 3G), resistin (Fig. 3H) release, reactive oxygen species production (Fig. 3J), but down-regulated adiponectin (Fig. 3I) secretion in a dose-dependent manner at concentrations of $0.1,1$ and $10 \mathrm{nmol} / \mathrm{L}$. Thus, AIF-1 potently stimulated reactive oxygen species production, inflammatory factors TNF $\alpha$, IL6 secretion, provoked resistin, but suppressed adiponectin release.

\section{The effect of AIF-1 on PPAR $\gamma$ expression and $N F-\kappa B$ phosphorylation}

To investigate the underlying mechanism of AIF-1 inducing inflammatory pathways, the expression of PPAR $\gamma$ and the phosphorylation of NF- $\kappa$ B were analyzed by Western blot. As shown in Fig. 4, AIF-1 over-expression inhibited PPAR $\gamma$ expression $(0.64 \pm 0.01$ fold, $P$

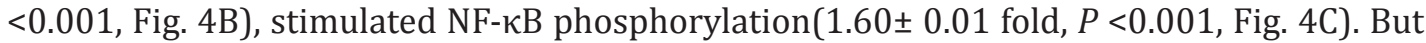
siAIF1-2 increased PPAR $\gamma$ expression (1.91 0.02 fold, $P<0.001$, Fig. 4B), suppressed NF- $\kappa \mathrm{B}$ activation (0.43 0.02 fold, $P<0.001$, Fig. 4C). Agree with this, AIF-1 protein down-regulated PPAR $\gamma$ expression to $0.85 \pm 0.03$ fold, $P<0.05$ at concentration of $0.1 \mathrm{nmol} / \mathrm{L}, 0.57 \pm 0.04$ fold, $P<0.01$ at concentration of $1 \mathrm{nmol} / \mathrm{L}$ and $0.35 \pm 0.07$ fold, $P<0.001$ at concentration of $10 \mathrm{nmol} / \mathrm{L}$ (Fig. 4E). It activated NF- $\mathrm{BB}$ to $1.31 \pm 0.03$ fold, $P<0.01$ at concentration of 0.1 $\mathrm{nmol} / \mathrm{L}, 1.89 \pm 0.02$ fold, $P<0.001$ at concentration of $1 \mathrm{nmol} / \mathrm{L}, 2.13 \pm 0.04$ fold, $P<0.001$ at 
concentration of $10 \mathrm{nmol} / \mathrm{L}$ (Fig. 4F). These results suggested that AIF-1disrupted PPAR $\gamma$ expression and provoked NF- $\mathrm{KB}$ signaling.

\section{AIF-1 mediates alteration of glucose consumption}

In addition, AIF-1 up-regulation led to a reduction in glucose consumption $(4.86 \pm 0.16$ $\mathrm{mmol} / \mathrm{L}$ remained vs $3.62 \pm 0.15 \mathrm{mmol} / \mathrm{L}$ remained in medium of normal pcDNA3.1 adipocytes and $5.21 \pm 0.16 \mathrm{mmol} / \mathrm{L}$ remained vs $4.15 \pm 0.08 \mathrm{mmol} / \mathrm{L}$ remained in medium of adipocytes with insulin resistance, $P<0.001$, Fig. 5A). While this effect was abolished by AIF-1 inhibition $(2.27 \pm 0.25 \mathrm{mmol} / \mathrm{L}$ remained vs $3.64 \pm 0.48 \mathrm{mmol} / \mathrm{L}$ remained in medium of normal pcDNA3.1 adipocytes, $P<0.001$, and $3.30 \pm 0.16 \mathrm{mmol} / \mathrm{L}$ remained vs $4.30 \pm 0.25$ $\mathrm{mmol} / \mathrm{L}$ remained in medium of adipocytes with insulin resistance, $P<0.01$, Fig. 5A). In accordance with this, AIF-1 protein also suppressed glucose uptake into 3T3L1 adipocytes $(2.10 \pm 0.03 \mathrm{mmol} / \mathrm{L}$ remained vs $1.49 \pm 0.01 \mathrm{mmol} / \mathrm{L}$ remained in medium of normal pcDNA3.1 adipocytes, $P<0.001$ and $4.52 \pm 0.03 \mathrm{mmol} / \mathrm{L}$ remained vs $4.28 \pm 0.02 \mathrm{mmol} / \mathrm{L}$ remained in medium of adipocytes with insulin resistance, $P<0.01$ at concentration of $0.1 \mathrm{nmol} / \mathrm{L}$; $3.22 \pm 0.05 \mathrm{mmol} / \mathrm{L}$ remained vs $1.49 \pm 0.01 \mathrm{mmol} / \mathrm{L}$ remained in medium of normal pcDNA3.1 adipocytes, $P<0.001$ and $4.89 \pm 0.04 \mathrm{mmol} / \mathrm{L}$ remained vs $4.28 \pm 0.02 \mathrm{mmol} / \mathrm{L}$ remained in medium of adipocytes with insulin resistance, $P<0.001$ at concentration of $1 \mathrm{nmol} / \mathrm{L}$; $4.71 \pm 0.04 \mathrm{mmol} / \mathrm{L}$ remained vs $1.49 \pm 0.01 \mathrm{mmol} / \mathrm{L}$ remained in medium of normal pcDNA3.1 adipocytes, $P<0.001$ and $5.06 \pm 0.02 \mathrm{mmol} / \mathrm{L}$ remained vs $4.28 \pm 0.02 \mathrm{mmol} / \mathrm{L}$ remained in medium of adipocytes with insulin resistance, $P<0.001$ at concentration of $10 \mathrm{nmol} / \mathrm{L})$. Therefore, AIF-1 impaired insulin-stimulated glucose uptake in adipocytes both under normal basal and insulin resistance conditions.

\section{Influence of AIF-1 on GLUT 4 tranlocation and Akt phosphorylation}

In the present study, AIF-1 over-expression reduced the content of GLUT 4 on the plasma membrane $(0.35 \pm 0.01$ fold in normal adipocytes, $P<0.01$; and $0.21 \pm 0.01$ fold in adipocytes with insulin resistance, $P<0.001$, Fig. 6B), while AIF-1 interference enhanced GLUT 4 translocation to the plasma membrane $(1.21 \pm 0.02$ fold in normal adipocytes, $P<0.05$; and $2.19 \pm 0.03$ fold in adipocytes

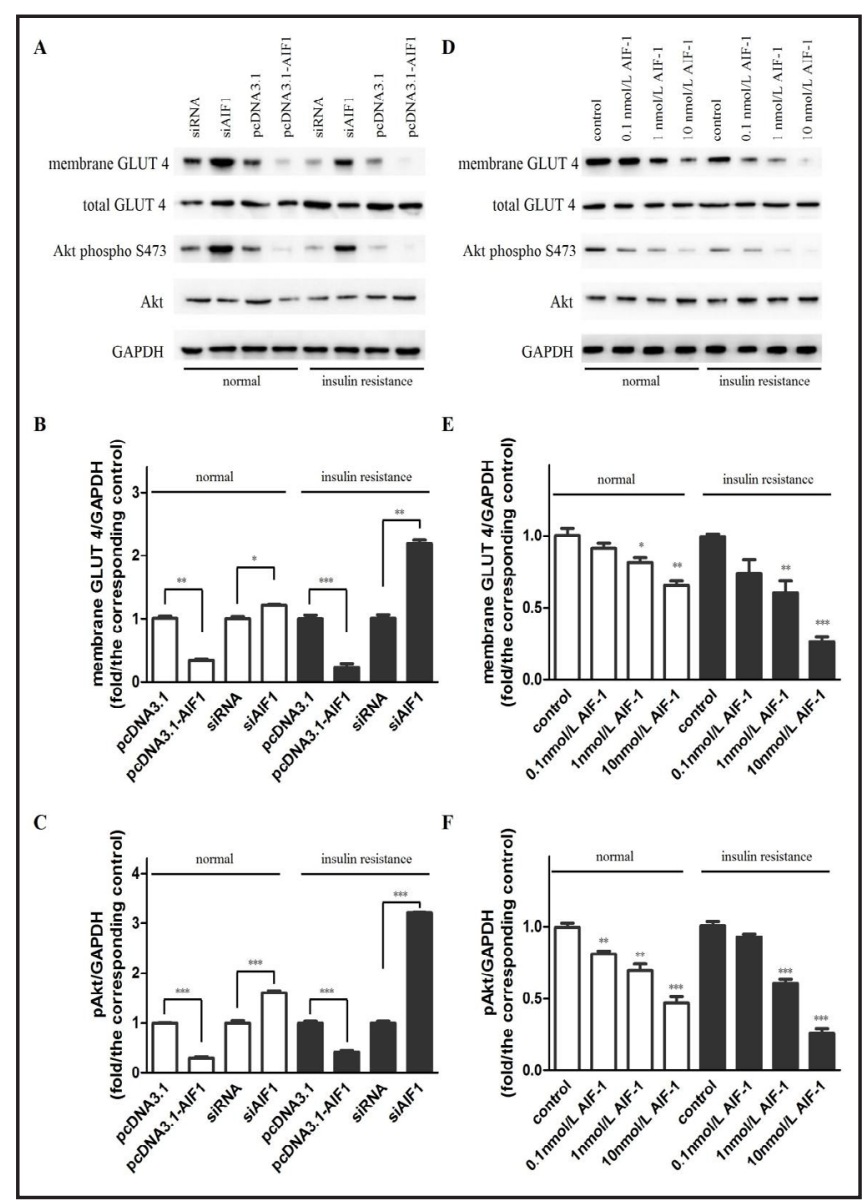

Fig. 6. The influence of AIF-1 on AKT phosphorylation and insulin-responsive glucose transporter 4 (GLUT 4) translocation to plasma membrane. 3T3L1 adipocytes were incubated in macrophage-conditioned medium $(\mathrm{A}, \mathrm{B}, \mathrm{C})$ or $0,0.1,1,10$ nmol/L AIF-1 in DMEM (D, E, F) supplemented with $10 \%$ fetal bovine serum for 2 days. The levels of membrane GLUT 4 and phosphorylated Akt (Ser473) were measured by Western blot. Data were the mean \pm SEM of three independent experiments. $\left({ }^{*} \mathrm{P}<0.05,{ }^{* *} \mathrm{P}<0.01,{ }^{* * *} \mathrm{P}<0.001\right)$. 


\section{Cellular Physiology Cell Physiol Biochem 2018;47:403-413 \\ and Biochemistry Published online: May 22, $2018 \quad$\begin{tabular}{l|l} 
DOI: 10.1159/000489952 & $\begin{array}{l}\text { C } 2018 \text { The Author(s). Published by S. Karger AG, Basel } \\
\text { www.karger.com/cpb }\end{array}$
\end{tabular} \\ Ren et al.: Mediates Insulin Signaling in Adipocytes}

with insulin resistance, $P<0.01$, Fig. 6B). Furthermore, AIF-1 significantly decreased insulinstimulated phosphorylation of Akt at Ser473 compared with controls $(0.28 \pm 0.03$ fold in normal adipocytes, $P<0.001 ; 0.46 \pm 0.02$ fold in adipocytes with insulin resistance, $P<0.001$, Fig. 6C), and AIF-1 expression down-regulation induced the phosphorylation $(1.53 \pm 0.02$ fold in normal adipocytes, $P<0.001 ; 3.24 \pm 0.05$ fold in adipocytes with insulin resistance, $P<$ 0.001 , Fig. 6C). Consistent with this, $0.1,1$ and $10 \mathrm{nmol} / \mathrm{L}$ AIF-1 protein also down-regulated the level of GLUT 4 on the plasma membrane and Akt phosphorylation in a concentration dependent manner. Impaired GLUT 4 translocation and Akt phosphorylation by AIF-1 may contribute to reduction of insulin-stimulated glucose consumption in adipocytes.

\section{Discussion}

Obesity is associated with macrophage accumulation in adipose tissue [3]. The macrophage-adipocyte crosstalk in obesity affects adipose tissue biology, but the molecular mechanisms and the key mediators remain largely unknown. In the present study, we investigated the effect of macrophage-derived AIF-1 on lipid storage, inflammatory responses and insulin sensitivity in adipocytes using two macrophage cell lines. One over-expressed AIF-1 via transfection of AIF-1 gene into RAW 264.7 cells, and the other decreased the constitutive expression of AIF-1 via transfection of targeting siRNA. We observed short term AIF-1 treatment slightly increased the amount of triglycerides on the $6^{\text {th }}$ day of differentiation, but cell apoptosis was not affected by AIF-1(data not shown).

Hypertrophied and dysfunctional adipocytes from obese subjects secrete several proinflammatory cytokines such as TNF- $\alpha$ and IL-6. It is well known that TNF- $\alpha$ positively contributes to the inflammatory adipocyte micro-environment, mainly by activating the classic transcription factor NF- $\mathrm{KB}$ signaling, the early key event in the pathogenesis of insulin resistance [24, 25]. Furthermore, it may also inhibit PPAR $\gamma$ through activation of NF- $\kappa B$ [26]. Since the GLUT 4 promoter contains response elements for, or regulated by PPAR $\gamma$ [27], it is likely that TNF- $\alpha$ could suppress GLUT 4 expression via a PPAR $\gamma$ dependent mechanism. On the other hand, elevated IL6 level is also associated with the development of insulin resistance via reduction of GLUT 4, PPAR $\gamma$ and insulin-stimulated glucose uptake in 3T3-L1 adipocytes [28]. Thus, the activation of inflammatory-linked pathways in adipocytes is an important mediator of insulin resistance. In addition, oxidative stress links inflammation to insulin resistance and intracellular reactive oxygen species in adipocytes result in insulin resistance through attenuation of insulin signaling [29]. In this study, AIF-1 induced reactive oxygen species production, TNF- $\alpha$ and IL6 secretion from 3T3L1 adipocytes, further suppressed PPAR $\gamma$ expression and activated NF- $\kappa B$, which may lead to insulin resistance in adipocytes.

Adiponectin is an adipocyte-secreted polypeptide with a principal role in the suppression of the metabolic derangements that may result in insulin resistance [30]. In contrast to adiponectin, the level of resistin has been shown to be up-regulated in subjects with insulin resistance [31]. The present experiment demonstrated that AIF-1 induced resistin secretion, while deduced adiponectin release from 3T3L1 adipocytes, which also implied the association of AIF-1 with insulin resistance.

LorenteCebrián and colleagues demonstrated AIF-1 was inversely correlated with insulin sensitivity as estimated by insulin tolerance test, and positively correlated with insulin resistance as assayed by homeostasis model assessment for insulin resistance [20], which inspired us to further investigate the direct inhibitory effect of AIF-1 on the insulin signaling in adipocytes. We observed that AIF-1 indeed reduced insulin-stimulated glucose uptake in 3T3L1 adipocytes both under normal basal and insulin resistance conditions. It is well known that insulin decreases blood glucose levels by promoting glucose uptake into adipose and muscle cells. Under basal conditions, about 95\% of GLUT 4 protein is localized within intracellular membrane compartments, with the remaining 5\% protein at the cell surface [32]. By contrast, insulin triggers GLUT 4 translocation to the plasma membrane, which results in glucose uptake [33]. It is worthy to note that impaired GLUT 4 translocation 


\section{Cellular Physiology Cell Physiol Biochem 2018;47:403-413 and Biochemistry Published online:May 22, $2018 \quad$\begin{tabular}{l|l} 
DOI: 2018 The Author(s). Published by S. Karger AG, Basel \\
www.karger.com/cpb
\end{tabular} \\ Ren et al.: Mediates Insulin Signaling in Adipocytes}

to the plasma membrane has been considered as one of the earliest defects leading to insulin resistance and type 2 diabetes [33]. In this study, AIF-1 impaired GLUT 4 translocation to plasma membrane. Next, it suppressed insulin-signal-transduction related molecule Akt phosphorylation. The protein kinase Akt is also known as protein kinase B (PKB). Upon activation, Akt is transported to the plasma membrane where it is phosphorylated at Thr-308 and Ser-473. Thereafter, Akt phosphorylates a range of substrates and the phosphorylation of these substrates orchestrates a complex metabolic program involving binding of insulin to the insulin receptor and the translocation of the glucose transporter GLUT 4 to the plasma membrane leading to glucose uptake [34].

In conclusion, our data suggest that macrophage-derived AIF-1 stimulated reactive oxygen species production, enhanced adipokine TNF $\alpha$, IL6, resistin secretion, decreased adiponectin release from 3T3L1 adipocytes probably via inducing NF- $\kappa \mathrm{B}$ activation, inhibiting PPAR $\gamma$ expression. Furthermore, it suppressed insulin-stimulated glucose uptake by depressing GLUT 4 translocation to plasma membrane and Akt phosphorylation.

\section{Acknowledgements}

This work was supported by the Natural Science Foundation of China (No. 31400794, ,31672395), National Key R\&D Program of China (2017JYD0501101) and Fundamental Research Funds for the Central Universities, Southwest University for Nationalities (2018NZD14).

\section{Disclosure Satement}

No conflict of interests exists.

\section{References}

1 King D: Foresight report on obesity. Lancet 2007;370:1754.

2 Wellen KE, Hotamisligil GS: Obesity-induced inflammatory changes in adipose tissue. J Clin Invest 2003;112:1785-1788.

- W Weisberg SP, McCann D, Desai M, Rosenbaum M, Leibel RL, Ferrante AW Jr: Obesity is associated with macrophage accumulation in adipose tissue. J Clin Invest 2003;112:1796-1808.

4 Lumeng CN, Saltiel AR: Inflammatory links between obesity and metabolic disease. J Clin Invest 2011;121:2111-2117.

5 Constant VA, Gagnon A, Landry A, Sorisky A: Macrophage-conditioned medium inhibits the differentiation of 3T3-L1 and human abdominal preadipocytes. Diabetologia 2006;49:1402-1411.

-6 Odegaard JI, Chawla A: Pleiotropic actions of insulin resistance and inflammation in metabolic homeostasis. Science 2013;339:172-177.

-7 Lacasa D, Taleb S, Keophiphath M, Miranville A, Clement K: Macrophage-secreted factors impair human adipogenesis: involvement of proinflammatory state in preadipocytes. Endocrinology 2007;148:868-877.

$>8$ Zhao YY, Yan DJ, Chen ZW: Role of AIF-1 in the regulation of inflammatory activation and diverse disease processes. Cellular Immunology 2013;284:75-83.

-9 Utans U, Arceci RJ, Yamashita Y, Russell ME: Cloning and characterization of allograft inflammatory factor-1: a novel macrophage factor identified in rat cardiac allografts with chronic rejection. J Clin Invest 1995;95:2954-2962.

10 Chen ZW, Ahen B, Ösenson CG, Cintra A, Bergman T, Moller C, Fuxe K, Mutt V, Jornvall H, Efendic S: Identification, isolation, and characterization of daintain (allograft inflammatory factor 1), a macrophage polypeptide with effects on insulin secretion and abundantly present in the pancreas of prediabetic BB rats. Proc Natl Acad Sci 1997;94:13879-13884.

-11 Imai Y, Ibata I, Ito D, Ohsawa K, Kohsaka S: A novel gene iba1 in the major histocompatibiliy complex class III region encoding an EF hand protein expressed in a monocytic lineage. Biochem Biophys Res Commun 1996;224:855-862.

12 Tanaka S, Suzuki K, Watanabe M, Matsuda A, Tone S, Koike T: Up-regulation of a new microglial gene, mrf1, in response to programmed neuronal cell death and degeneration. J Neurosci 1998;18:6358-6369. 


\section{Cellular Physiology Cell Physiol Biochem 2018;47:403-413 \begin{tabular}{lll} 
DOI: 10.1159/000489952 & and Biochemistry & $\begin{array}{l}\text { O } 2018 \text { The Author(s). Published by S. Karger AG, Basel } \\
\text { www.karger.com/cpb }\end{array}$ \\
\cline { 2 - 3 }
\end{tabular} \\ Ren et al.: Mediates Insulin Signaling in Adipocytes}

13 Yang ZF, Ho DW, Lau CK, Lam CT, Lum CT, Poon RT, Fan ST: Allograft inflammatory factor-1 (AIF-1) is crucial for the survival and pro-inflammatory activity of macrophages. Int Immunol 2005;17:1391-1397.

14 Tian Y, Kelemen SE, Autieri MV: Inhibition of AIF-1 expression by constitutive siRNA expression reduces macrophage migration, proliferation, and signal transduction initiated by atherogenic stimuli. Am J Physiol Cell Physiol 2006;290:C1083-C1091.

15 Sommerville LJ, Kelemen SE, Ellison SP, England RN, Autieri MV: Increased atherosclerosis and vascular smooth muscle cell activation in AIF-1 transgenic mice fed a high-fat diet. Atherosclerosis 2012;220:45-52.

-16 Zhao YY, Huang XY, Chen ZW: Daintain/AIF-1 (Allograft Inflammatory Factor-1) accelerates type 1 diabetes in NOD mice. Biochem Biophys Res Commun 2012;427:513-517.

-17 Deininger MH, Weinschenk T, Meyermann R, Schluesener HJ: The allograft inflammatory factor-1 in Creutzfeldt-Jakob disease brains. Neuropathol Appl Neurobiol 2003;29:389-399.

-18 Thorleifsson G, Walters GB, Gudbjartsson DF, Steinthorsdottir V, Sulem P, Helgadottir A, Styrkarsdottir U, Gretarsdottir S, Thorlacius S, Jonsdottir I, Jonsdottir T, Olafsdottir EJ, Olafsdottir GH, Jonsson T, Jonsson F, Borch-Johnsen K, Hansen T, Andersen G, Jorgensen T, Lauritzen T, Aben KK, Verbeek AL, Roeleveld N, Kampman E, Yanek LR, Becker LC, Tryggvadottir L, Rafnar T, Becker DM, Gulcher J, Kiemeney LA, Pedersen O, Kong A, Thorsteinsdottir U, Stefansson K: Genome-wide association yields new sequence variants at seven loci that associate with measures of obesity. Nat Genet 2009;41:18-24.

19 Casimiro I: Role of allograft inflammatory factor-1 (Aif-1) in macrophage activity, atherosclerosis, and obesity. New York: Yeshiva University 2010;3430518.

-20 Lorente-Cebrián S, Decaunes P, Dungner E, Bouloumié A, Arner P, Dahlman I: Allograft inflammatory factor 1 (AIF-1a new human adipokine involved inadipose inflammation in obese women. BMC Endocr Disord 2013;13: 54.

-21 Ye F, Than A, Zhao YY, Goh KH, Chen P: Vesicular storage, vesicle trafficking, and secretion of leptin and resistin: the similarities, differences, and interplays. J Endocrinol 2010;206:27-36.

22 Rizzatti V, Boschi F, Pedrotti M, Zoico E, Sbarbati A, Zamboni M: Lipid droplets characterization in adipocyte differentiated 3T3-L1 cells: size and optical density distribution. Eur J Histochem 2013;e24

23 Yin J, Hu R, Chen M, Tang J, Li F, Yang Y, Chen J: Effects of berberine on glucose metabolism in vitro. Metabolism 2002;51:1439-1443.

24 Matsui Y, Tomaru U, Miyoshi A, Ito T, Fukaya S, Miyoshi H, Atsumi T, Ishizu A: Overexpression of TNF- $\alpha$ converting enzyme promotes adipose tissue inflammation and fibrosis induced by high fat diet. Exp Mol Pathol 2014;97:354-358.

-25 Ruan H, Pownall HJ, Lodish HF: Troglitazone antagonizes tumor necrosis factor-alpha-induced reprogramming of adipocyte gene expression by inhibiting the transcriptional regulatory functions of NFkappaB. J Biol Chem 2003;278:28181-28192.

26 Jain RG, Phelps KD, Pekala PH: Tumor necrosis factor-alpha initiated signal transduction in 3T3-L1 adipocytes. J Cell Physiol 1999;179: 58-66.

-27 Marín-Juez R, Diaz M, Morata J, Planas JV: Mechanisms regulating GLUT4 transcription in skeletal muscle cells are highly conserved across vertebrates. PLoS One 2013;8:e80628.

-28 Rotter V, Nagaev I, Smith : Interleukin-6 (IL-6) induces insulin resistance in 3T3-L1adipocytes and is, like IL-8 and TNF- $\alpha$, overexpressed in human fat cells from insulin-resistant subjects. J Biol Chem 2003;278:45777-45784.

29 Bondia-Pons I, Ryan L, Martinez JA: Oxidative stress and inflammation interactions in human obesity. J Physiol Biochem 2012;68:701-711.

-30 Li S, Shin HJ, Ding EL, van Dam RM: Adiponectin levels and risk of type 2 diabetes: a systematic review and meta-analysis. JAMA 2009;302:179-188.

-31 Chen BH, Song Y, Ding EL, Roberts CK, Manson JE, Rifai N, Buring JE, Gaziano JM, Liu S: Circulating levels of resistin and risk of type 2 diabetes in men and women: results from two prospective cohorts. Diabetes Care 2009;32:329-334.

-32 Fujita H, Hatakeyama H, Watanabe TM, Sato M, Higuchi H, Kanzaki M: Identification of three distinct sites of insulin-mediated GLUT4 trafficking in adipocytes using quantitative single molecule imaging. Mol Biol Cell 2010;21:2721-2731.

-33 Shepherd PR, Kahn BB: Glucose transporters and insulin action--implications for insulin resistance and diabetes mellitus. N Engl J Med 1999;341:248-257.

34 Manning BD, Cantley LC: AKT/PKB signaling: navigating downstream. Cell 2007;129:1261-1274. 